Running Head: ATTACHMENT, ER, AND ADJUSTMENT

\title{
Common and Distinct Correlates of Depression, Anxiety, and Aggression: Attachment and Emotion Regulation of Sadness and Anger
}

\author{
Sarah J. Clear \\ Alex A. Gardner \\ Haley J. Webb \\ Melanie J. Zimmer-Gembeck \\ School of Applied Psychology, Griffith University
}

Conflict of Interest: Author A declares that she has no conflict of interest. Author B declares that he has no conflict of interest. Author C declares that she has no conflict of interest. Author D declares that she has no conflict of interest.

Key words : Attachment, emotion, anxiety, depression, aggressive behaviour, emerging adulthood. 
Corresponding author:

Sarah J. Clear Griffith University

School of Applied Psychology

Parklands Dr, G40_7.86

Southport QLD 4222

Australia

Tel: +61 756789085

FAX: +61 756788291

sarah.clear@griffithuni.edu.au

Dr Haley J Webb

Griffith University

School of Applied Psychology

Parklands Dr, G40_7.86

Southport QLD 4222

Australia

Tel: +61 756789085

FAX: +61 756788291

haley.webb@griffithuni.edu.au
Alex A Gardner

Griffith University

School of Applied Psychology

Parklands Dr, G40_7.86

Southport QLD $42 \overline{2} 2$

Australia

Tel: +61 756789085

FAX: +61 756788291

alex.gardner@griffithuni.edu.au

Prof Melanie J Zimmer-Gembeck

Griffith University

School of Applied Psychology

Parklands Dr, G40_7.86

Southport QLD $42 \overline{2} 2$

Australia

Tel: +61 756789085

FAX: +61756788291

m.zimmer-gembeck@griffith.edu.au

\section{Authors Contributions}

SC conceived of the study, participated in its design and coordination, conducted data collection, interpreted the data, and drafted the manuscript; AG drafted the manuscript and critically revised the manuscript; HW performed the statistical analysis and helped draft the manuscript. MZG was the overall supervisor of the project, concieved of the study, performed the statistical analysis and interpretation of the data, and drafted the manuscript. All authors read and approved the final manuscript.

\section{Acknowledgments}

We would like to thank Shawna Campbell for her assistance with data collection. 


\begin{abstract}
Drawing from attachment and emotion theories, we tested a model whereby emerging adults' anxious and avoidant attachment would have specific associations with dysregulation and suppression of sadness and anger and would be unique correlates of emotional and behavioral problems. Participants were 383 (47\% men) students between 16 and 23 years $(M=19.6, S D=$ 1.58) who completed a questionnaire to assess attachment, emotion dysregulation and suppression, depressive and social anxiety symptoms, and aggressive behavior. In a path model, greater anxious attachment was associated with more emotion dysregulation, whereas greater avoidant attachment was associated with greater emotion suppression. Greater sadness dysregulation was uniquely and significantly associated with depression and social anxiety but not aggression, whereas greater anger dysregulation was associated with aggressive behavior but not depression and anxiety. Also, participants with elevated attachment insecurities reported heightened emotional and behavioral problems. Anxious attachment had the most pervasive impact on all forms of symptoms, either directly or indirectly via emotion dysregulation. Yet, there was also evidence that a focus on regulation of sadness, relative to anger, identified unique links with depression and social anxiety, relative to aggressive behavior.
\end{abstract}




\section{Common and Distinct Correlates of Depression, Anxiety, and Aggression: Attachment and Emotion Regulation of Sadness and Anger}

In classic attachment theory, early childhood experiences with caregiving are described as the foundations for the formation of a general working model of relationships, which is also sometimes referred to as attachment orientation or attachment style (Bowlby, 1969, 1973, 1980; Cassidy, 1994). By adolescence and into adulthood, the working model has been described as a general conception of the mental representations of the self (e.g., beliefs about one's worthiness) and others (e.g., expectations about the availability and responsiveness of others) (Mikulincer \& Shaver, 2007, 2012). Along with the development of a working model, early interactions, which include caregivers' assisting children in their nascent attempts to regulate emotions and, later, coregulation and support in self-regulation, are also described as essential for the later formation of intra-individual emotion regulation (ER) skills and competencies (Brumariu, 2015; Brumariu \& Kerns, 2013; Kobak, Cassidy, Lyons-Ruth, \& Ziv, 2006; Kopp, 1989; Morris, Silk, Steinberg, Myers, \& Robinson, 2007; Skinner \& Zimmer-Gembeck, 2016; Zimmer-Gembeck \& Skinner, 2016; Zimmer-Gembeck et al., 2017). Thus, attachment and the subsequent regulatory capacities to manage (express) emotional reactions would be expected to covary with each other, since each have a foundation in the early caregiving environment. It would also be expected that attachment and ER would co-vary with each other even beyond childhood, despite later experiences and advances. In support of this notion, attachment orientation has been found to account for some of the individual differences in how toddlers, children, adolescents and adults manage and regulate their emotions (Malik, Wells, \& Wittkowski, 2015; Mikulincer \& Shaver, 2007; Wei, Vogul, Ku, \& Zakalik, 2005), with individuals classified as insecure showing more maladaptive ER and those classified as secure having ER advantages (Brumariu, 2015; Brumariu \& Kerns, 2013; 
Kerns, 2008; Marganska, Gallagher, \& Miranda, 2013; Lyons-Ruth \& Jacobvitz, 2008; ZimmerGembeck et al., 2017).

Much of this emphasis on attachment and ER, and their interrelation, has been informative for identifying individuals who are on a maladaptive mental health or social trajectory (Brenning, Soenens, Braet, \& Bosmans, 2012; Clear \& Zimmer-Gembeck, 2017; Kobak et al., 2006; Mikulincer \& Shaver, 2007; Wei, Heppner, \& Mallinckrodt, 2003). Studies have shown that insecure attachment and ER are independently associated with symptoms of depression, social anxiety, and behavior problems (Brenning \& Braet, 2013; Brenning et al., 2012; Mikulincer \& Shaver, 2007; Mikulincer, 1998; Viana \& Rabian, 2008). Further Brenning and Braet (2013) pioneered the investigation of regulating specific emotions and tested a model of the associations between adolescents' attachment, ER of specific emotions, and psychological problems. The aim of the current study was to examine these associations among emerging adults, focusing on associations between insecure (anxious and avoidant) attachment orientation, and depressive symptoms, social anxiety symptoms and aggressive behavior. Moreover, a second aim was to test the mediating role of ER of sadness and anger in these associations.

The present study was novel because of the focus on emerging adults, a time of life when ER strategies are becoming increasingly mature but may not yet have reached their optimal level (Aldao, Nolen-Hoeksema, \& Schweizer, 2010; Haga, Kraft, \& Corby, 2009; Skinner \& ZimmerGembeck, 2007). Emerging adulthood is a time when there is a rapid escalation in autonomy from parents and an increased reliance on friendships and romantic relationships for support and information (Sieffge-Krenke, 2011). Thus, the likelihood of new interpersonal stressors occurring during emerging adulthood is quite high, which can uniquely challenge working models of attachment and ER capacity, resulting in even stronger links of attachment and ER 
with symptoms of internalizing and externalizing disorders. We could locate no previous study that has examined associations between attachment, emotion-specific ER, and symptoms of psychopathology among emerging adults.

\section{Attachment Orientation, ER, and Symptoms}

There are many reasons to anticipate that anxious and avoidant attachment orientations would be associated with ER, but this association may depend on the specific form, aspect, or emotion of the regulation strategies under examination. In particular, studies have found that anxious and avoidant attachment can interfere with two forms of ER, including heightened signs of dysregulation and more use of suppression, but that this would differ depending on whether the focus is on anxious or avoidant attachment (Mikulincer \& Shaver, 2007). Individuals higher in an anxious attachment orientation have been described as hyperactivating, whereby they emotionally overreact and attempt to elicit increased attention from others when distressed (Wei et al., 2005). In other words, individuals higher in anxious attachment would display more negative emotions and, often excessively, seek closeness to others when experiencing stress. They would worry more intensely about the availability of the attachment figure (or close others) for support and soothing (Brenning \& Braet, 2013). This implies particular challenges with heightened emotion dysregulation.

Individuals with a more prominent avoidant orientation are described as deactivating, whereby they suppress negative emotions and distance themselves from others when distressed (Mikulincer, Shaver, \& Pereg, 2003). Deactivation implies that those with avoidant tendencies would be more likely to respond to problems by discounting the severity of their feelings, distancing themselves from others when distressed, and less often seeking or giving social support (Wei et al., 2005). Individuals high in avoidant attachment do not feel as comfortable 
depending on others, have a strong preference for emotional distance, and they minimise their generalised distress (Cassidy, 1994). Thus, their ER pattern may be marked by more suppression of emotion in order to reduce displays that will evoke others' support. Further, emotion theories suggest ER may vary as a function of specific emotion type. For example, the differential emotions perspective where each emotion serves an adaptive social function or provokes a particular reaction from others (Izard, 1991), along with the functionalist theory of emotion suggesting specific emotions have specific social and relationship functions (e.g., Campos, Mumme, Kermoian, \& Campos, 1994). Considering different social preferences among attachment orientations (i.e., emotional closeness or distance), and distinct emotions serving particular social or relational functions, we might expect ER of specific emotions to vary between individuals with different attachment orientations.

Considering both ER strategies and insecure attachment orientation may help to better account for symptoms of psychopathology than examining either in isolation. Most, if not all, forms of psychopathology involve restricted capacity for adaptive emotion recognition, expression or regulation, and maladaptive responses to negative emotions (Compas, ConnorSmith, Saltzman, Thomsen, \& Wadsworth, 2001; Southam-Gerow \& Kendall, 2002). Therefore, ER is proposed as an explanation for when and why attachment orientation has such wideranging effects on adjustment, adaption and psychopathology (e.g., see Brenning et al., 2012; Contreras, Kerns, Weimer, Gentzler, \& Tomich, 2000; Kullik \& Petermann, 2013; Morris et al., 2007; Wei et al., 2003). In particular, the ER strategies that are more likely to be found among individuals higher in attachment insecurity are most likely to interfere with adaptive responding to stress that will boost the development of symptoms of psychopathology in all forms (Mikulincer, et al., 2003). To understand how attachment anxiety and avoidance may be 
associated with symptoms of depression, social anxiety, and behavior problems, however, it is useful to consider the specific emotions that are being regulated and how they may or may not be managed. In particular, difficulties with regulating sadness will likely be more prominent for individuals who exhibit more depressive and anxious symptoms, whereas difficulties regulating anger will likely be more prominent for individuals who exhibit more aggressive behavior (Zimmer-Gembeck, Nesdale, Webb, Khatibi, \& Downey, 2016). Taken together, the attachment model of ER suggests that anxious versus avoidant attachment should identify individuals who are more likely to dysregulate or suppress emotions, in turn give an indication to the types of pathologies that may be experienced. However, previous studies have shown mixed evidence in supporting the pattern of suppression for individuals higher in attachment avoidance as related to emotional or behavioral problems in various child, adolescent, and adult samples (Malik et al., 2015; Marganska et al., 2013). We however posit that the particular emotion that is dysregulated or suppressed should help to better account for when and why each form of attachment is associated with depression, social anxiety, and aggressive behavior.

\section{The Current Study}

ER may be challenging for those who report heightened attachment insecurity (Mikulincer \& Shaver, 2007), and ER deficits play a prominent role in many forms of psychopathology (Compas et al., 2001; Southam-Gerow \& Kendall, 2002). Furthermore, this period of development is a significant transition where the onset of several interpersonal or psycho-social stressors may place additional strain on emerging adults' ER capacities (SieffgeKrenke, 2011; Skinner \& Zimmer-Gembeck, 2016). In the present study, we extended previous research by examining the unique pathways of vulnerability that anxious and avoidant attachment may confer on symptoms of depression, social anxiety, and aggression through, ER 
(dysregulation and suppression) of sadness and anger during the period of emerging adulthood. The following hypotheses were tested:

H1 - Greater sadness and anger dysregulation will be associated with greater anxious attachment, given that individuals higher in anxious attachment strive to preserve or enhance closeness to others.

H2- Greater suppression of sadness and anger will be associated with greater avoidant attachment, given that individuals higher in avoidant attachment prefer emotional distance from others and self-reliance.

H3 - Greater sadness dysregulation will be uniquely associated with depressive and social anxiety symptoms.

H4 - Greater anger dysregulation will be uniquely associated with aggressive behavior. H5 - Depressive and social anxiety symptoms and aggressive behavior will be associated with greater anxious attachment via emotion dysregulation (especially sadness dysregulation), given the significant role of sadness in emotional problems. Note that we also tested the associations of avoidant attachment with all three outcomes via sadness and anger suppression, but made no specific hypotheses because of the mixed evidence in the literature supporting this indirect association.

\section{Method}

\section{Participants}

The 383 participants (181 young men, 202 young women) were undergraduate university students with a mean age of 19.6 years $(S D=1.58$; between the ages of 16 to 23$)$. Concerning family status, $68 \%$ of the participants were from intact families, whereas the remaining participants were from divorced or separated families, or families where one of the parents was 
deceased. Seventy percent of participants identified as being white/Caucasian, 19\% had an origin in Asia, 2\% identified as being Australian First People or Pacific Islander, while the remaining $9 \%$ identified as other.

\section{Procedure}

After receiving approval from the university Human Subjects Review Committee, participants were recruited from university campus common use areas $(87.5 \%)$ or from a pool of first year psychology students (12.5\%). Participants completed questionnaires under supervision of a researcher. First year psychology students received course credit for participation. All other participants received a chocolate bar after completing the questionnaire.

\section{Measures}

Attachment orientation. Participants completed the Experiences in Close Relationships Scale-Revised-General Short Form (ECR-R-GSF; Wilkinson, 2011), for the assessment of general relationship attachment anxiety and avoidance in adolescents and young adults. The anxiety scale (10 items) targets feelings of fear and abandonment and strong desires for closeness to others (e.g., "I find that other people don't want to be as close as I would like"). The avoidance scale (10 items) targets discomfort with closeness and dependence on others (e.g., "I prefer not to show others how I feel deep down"). Items were rated on a 5-point scale from 1 (strongly disagree) to 5 (strongly agree), Cronbach's $\alpha$ s were .86 and .79 for anxious and avoidant attachment, respectively.

Depressive symptoms. Participants completed 20 items from the Beck Depression Inventory II (BDI-II; Beck, Steer, \& Brown, 1996) to measure the severity of depression symptoms (one item referring to suicide was not included). For each item, participants chose one 
of four responses that best described him or her (e.g., "I feel sad much of the time"), Cronbach's $\alpha=.91$.

Social anxiety symptoms. Participants completed the social anxiety scale for adolescents (SAS-A) (La Greca \& Lopez, 1998) to measure the subjective experience of social anxiety. The scale contained 18 descriptive self-statements (e.g., "I worry about doing something new in front of others"). Each item was rated on a 5-point scale from 1 (not true) to 5 (very true) according to how much the item was true for the participant, Cronbach's $\alpha=.93$.

Aggressive behavior. Participants completed 17 items from the widely-used Youth Self Report (YSR: Achenbach \& Rescorla, 2001) to measure aggressive behavior (e.g., "I destroy things belonging to others"). Each item was rated on a 5-point scale from 1 (not true) to 5 (very true), Cronbach's $\alpha=.89$.

Sadness and anger dysregulation and suppression. Self-reported ER strategies were assessed using the dysregulation (six items, e.g., "I often behave in a stressful or anxious way, even if I don't want to behave like that") and suppression (seven items, e.g., "I try to ignore feelings of stress or anxiety") subscales of the ER inventory (Roth, Assor, Niemiec, Ryan, \& Deci., 2009). Items were adjusted to refer to emotions of sadness and anger. For example, the item "I try to ignore feelings of stress or anxiety" was changed to "I try to ignore feelings of sadness/anger.” Response options ranged from 1 (strongly disagree) to 5 (strongly agree).

Items were submitted to an exploratory factor analysis with an orthogonal rotation (varimax). Using the criterion of an eigenvalue $>1$, four factors were extracted, with items for the four proposed subscales of ER loading on the four factors. The factors accounted for $64 \%$ of the variance in the items. Sadness dysregulation items loaded on the first factor (eigenvalue $=$ 7.3, loadings from .69 to .84). Anger dysregulation items loaded on the second factor (eigenvalue 
$=5.0$, loadings from .71 to .80 ). Sadness suppression items loaded on the third factor (eigenvalue

$=2.5$, loadings from .64 to .79). Anger suppression items loaded on the fourth factor (eigenvalue $=1.7$, loadings from .54 to .85$)$. All crossloadings were less than .35 . Items loadings highly on each factor were averaged to form total scores, Cronbach's $\alpha$ was .91 and .87 for sadness suppression and dysregulation, and .89 and .88 for anger suppression and dysregulation, respectively.

\section{Overview of the Data Analyses}

After computing $M \mathrm{~s}, S D \mathrm{~s}$, $t$-tests to compare young men and women, and correlations between all measures, MPlus was used to test a concurrent path model. This model was fit to examine hypotheses pertaining to the direct effects of anxious and avoidant attachment on emotion-specific dysregulation and suppression, symptoms of depression and social anxiety, and aggressive behavior, as well as the indirect effects of attachment on symptoms and behaviors via ER. Based on bivariate correlations, significant associations between measures at each time point were freed (see Figure 1). To assess model fit, goodness-of-fit indices $\left(\chi^{2}, \chi^{2}\right.$ relative to sample size, comparative fit index - CFI, and root mean square error of approximation - RMSEA) were examined. For comparison to the primary model fit, we also fit models removing some or all paths from attachment to depression, social anxiety and aggressive behavior.

\section{Results}

\section{Correlations between Measures, Descriptive Statistics, and Sex Differences}

Tables 1 and 2 present correlations, $M \mathrm{~s}$ and $S D$ s, and $t$-tests to examine sex differences. As can be seen in Table 2, young women compared to men reported significantly higher depressive and social anxiety symptoms, less aggressive behavior, and more dysregulation of sadness. 


\section{A Model of Attachment, ER, Symptoms and Behavior}

Primary model. The model of attachment, ER, and symptoms (see Figure 1) had an excellent fit to the data, $\chi 2(1)=0.00, p=.99, \mathrm{CFI}=1.00, \mathrm{RMSEA}=.000(90 \% \mathrm{CI}=.000-.000)$, $p=1.00$, and explained $33 \%$ of the variance in depression, $42 \%$ of the variance in social anxiety, and $25 \%$ of the variance in aggressive behavior. In this model all paths were estimated, with the exclusion of one covariance between anger dysregulation and anger suppression, which was not significant.

Table 3 and Figure 2 present the standardized associations of attachment with ER (i.e., pathway A, Figure 1). Consistent with Hypotheses 1 and 2 and providing support for a differentiated pattern of associations between attachment and ER, attachment anxiety was uniquely and significantly associated with greater dysregulation of sadness and anger, whereas avoidant attachment was significantly associated with greater suppression of sadness and anger.

Table 4 presents the standardized direct, indirect and total associations of attachment and ER with depression, social anxiety and aggression (i.e., pathways B and C, Figure 1). See also Figure 2 provides an illustration of the direct associations of attachment and ER with depression, social anxiety and aggression. Supporting Hypothesis 3 and 4, greater sadness dysregulation was associated with more depressive and social anxiety symptoms, but was not significantly associated with aggressive behavior, whereas the opposite pattern was found for anger dysregulation. Providing support for Hypothesis 5, individuals higher in anxious attachment reported more symptoms of depression and social anxiety and more aggressive behavior directly and indirectly via emotion dysregulation. Finally, individuals higher in avoidant attachment reported more depressive and social anxiety symptoms, although avoidant attachment was not directly or indirectly associated with aggressive behavior. When indirect effects of attachment on 
symptoms and behavior via each measure of ER were examined (i.e., 24 individual indirect effects), two positive indirect effects stood out as significant: the indirect effect of anxious attachment on depressive symptoms via sadness dysregulation $(.14, p<.05)$, and the indirect effect of anxious attachment on aggressive behavior via anger dysregulation $(.15, p<.01)$.

Alternative models. When we removed all paths from attachment to symptoms and behavior, the fit of the model was less than adequate, $\chi 2(7)=130.31, p<.01, \mathrm{CFI}=.87$, RMSEA $=.215(90 \% \mathrm{CI}=.183-.248), p<=.01$. However, freeing the direct effects of anxious attachment on symptoms and aggressive behavior resulted in a good fitting model, $\chi 2(4)=$ $17.21, p=.002, \mathrm{CFI}=.99, \mathrm{RMSEA}=.093(90 \% \mathrm{CI}=.051-.140), p=.05$. Thus, the addition of the direct effects of anxious attachment on symptoms and behavior (but not the direct effects of avoidant attachment on symptoms and behavior) was sufficient to produce a good fitting model.

\section{Discussion}

Attachment theory suggests that an individuals' attachment orientation will be essential for the formation of ER capacities and the development of psychopathology symptoms (Brumariu, 2015; Brumariu \& Kerns, 2013; Kobak et al., 2006; Kopp, 1989; Morris et al., 2007; Zimmer-Gembeck et al., 2017). In the multivariate path model tested here, there was support for the hypothesized associations of emerging adults' attachment (anxious and avoidant), emotion dysregulation and emotion suppression with emotional and behavioral problems (i.e., depression, social anxiety and aggressive behavior). Emerging and young adults who reported heightened anxious and avoidant attachment also reported more dysregulation and suppression of sadness and anger, with anxious attachment uniquely relevant for dysregulation and avoidant attachment uniquely relevant for suppression. Moreover, those who reported more sadness dysregulation had elevated depressive and social anxiety symptoms, whereas those who reported more anger 
dysregulation had elevated aggressive behavior. Thus, consistent with past research (Mikulincer \& Shaver, 2007; Mikulincer et al., 2003; Wei et al., 2005), the results supported the distinctive roles of anxious versus avoidant attachment in emotion dysregulation versus suppression. The results also reveal the importance of considering each of sadness and anger dysregulation in emotional or behavioral symptoms (Brenning \& Braet, 2013). Moreover, anxious attachment had unique and direct associations with emotional and behavioral problems, while there was less support for the distinctive role of avoidant attachment for understanding aggressive behavior.

\section{Key Findings and Future Research Directions}

Attachment, regulation, symptoms and behavior. As hypothesized (Hypothesis 1), individuals who reported more anxious attachment also reported more dysregulation of emotion, including sadness and anger, and those who reported more avoidant attachment reported more suppression of emotion. These findings support Shaver and Mikulincer's (2002) description of the role of attachment working models in ER, whereby individuals who are more anxiously attached may have a tendency toward greater dysregulation of emotion because displays of these emotions may be useful for gaining support from others when distressed. These findings align with neopsychoanalytic perspectives that emphasize the goals of emotion regulation or dysregulation (Sroufe, 1996), whereby emotions and emotion regulation are functional for meeting goals or needs. With respect to our findings, anxious attachment is associated with heightened emotion dysregulation. This may be because individuals who endorse more anxious attachment do so because they have greater worries and concerns regarding the loss of intimacy with others and because greater intensity of emotional displays will likely draw attention from others and maintain some feeling of closeness (Sroufe, 1996; Wei et al., 2005). Also, as described in adult attachment theory (Mikulincer \& Shaver, 2007), individuals who are higher in 
avoidant attachment tend to suppress their emotions more so than others, which may be a strategy for maintaining distance from others, consistent with their tendency to avoid intimacy, even when they are distressed and support may be helpful.

Our findings are also consistent with Winnicott's (1965) theory of attachment and the development of the false self. Winnicott described how a false self can emerge when a developing infant is not nurtured in an environment where the 'good enough' parent mirrors, holds, and responds to emotional upsets. In these circumstances, a child can internalize a representation of the mother as emotionally unavailable, critical or possibly intrusive (Newman, 2013; Winnicott, 1965). Because this internalized object (i.e., the unavailable parent) has failed the child and provided its own source of emotional discomfort, over time, and across many poor interpersonal experiences, the false self serves the purpose; it protects the individual from further unsupportive emotional or interpersonal experiences. Therefore, our findings provide some support in the sense that when insecure attachment has developed, the false self (which may be integrated into the attachment internal working model) serves the purpose of protecting emerging and young adults' true self via their utilization of unhelpful ways for managing negative emotions to safeguard against perceived threat. Thus, it is not surprising that young people who reported higher insecure attachment reported greater ER-deficits, which in turn, according to both attachment theory (Bowlby, 1969; 1980) and Winnicott's (1965) view of the development of the false vs. true self, should also be associated with symptoms of psychopathology.

Consistent with the above expected links between attachment and symptoms of psychopathology, another set of associations tested in our model was the direct and indirect (via emotion dysregulation and suppression) associations of attachment with symptoms of depression, social anxiety and aggression. Our expectations here were partially supported. In 
particular, there was support for our hypothesis of a predominant role of anxious attachment and sadness dysregulation in depression and anxiety. We found that sadness dysregulation had associations with depression and social anxiety but not aggressive behavior, and anger dysregulation had an association with aggressive behavior but not depression and social anxiety. The focus on the regulation of two different emotions - sadness and anger - did facilitate an understanding of when dysregulation may coincide with different symptoms, and that anxious attachment may directly impact on symptoms but also may have an indirect association via its association with greater dysregulation of emotion. As suggested by Emde (1980), this may be the culmination of a developmental pattern whereby emotion dysregulation, and the heightened emotional displays of sadness or anger that come with dysregulation, bring others closer or keep others at a distance. This pattern of dysregulation, emotion displays and associated behaviors increases the likelihood of following a trajectory marked by unmet intimacy and relationship goals, escalating distress, and symptoms of psychopathology.

Our findings were less clear for avoidant attachment and aggressive behavior, whereby we found no significant unique association between them (either direct or indirect). Instead, it was individuals who reported more anxious attachment, and not avoidant attachment, that reported more aggressive behavior. Further, avoidant attachment had small (but significant) associations with a higher level of depressive and social anxiety symptoms. These findings might be partly explained by what we did find regarding the unique associations of anxious and avoidant attachment with ER; these associations did not divide along the form of emotion - sad or angry - rather, they divided along the ER deficit - dysregulation or suppression, as predicted. This suggests that anxious attachment may be an indicator of dysregulation across a range of emotions, which accounts for its widespread direct and indirect (via ER) impact on all three 
forms of emotional and behavioral problems. In contrast, avoidant attachment is more of an indicator of a greater reliance on emotional suppression, which could explain its rather weak links with all forms of problems measured here as greater emotion suppression would inhibit the ability to report problems. Despite not being completely consistent with our hypotheses, the findings are consistent with some past research, which has shown that both dependent (i.e., anxious) and avoidant interpersonal styles in emerging adults are associated with social anxiety in relationships (Darcy, Davila, \& Beck, 2005). Moreover, other studies have also found that individuals who are avoidant tend to report high levels of anxiety and depression, due to low levels of trust within relationships and an increased tendency to distance themselves from support (Marganska et al., 2013; Muris, Meesters, van Melick, \& Zwambag, 2001).

Emotional suppression and dysregulation. It is not clear why emotional suppression did not play more of a role in symptoms in the present study. We did find fairly strong associations of avoidant attachment with sadness suppression. Yet, these associations appear weaker when compared to the conclusions of a past meta-analysis, which reported medium to large effects sizes for suppression with forms of psychopathology in adulthood (Aldao et al., 2010). One possibility is, given that most past studies have asked about suppression of "emotion" or "distress" in general, by focusing individuals on specific emotions in the present study, we might have identified that it is not suppression of the specific emotions of sadness or anger that is associated with heightened symptomology, but rather a tendency to suppress a broad range of feelings. Another possibility is the age of the participants. It is possible that self-reliance, which may be exhibited among those higher in avoidant attachment, is not as problematic for symptom development in emerging or early adulthood as compared to among older adults (Mikulincer at al., 2003). Emerging and young adulthood is often prior to the formation of committed 
relationships outside the home. For this age group, parents may still be significant sources of support, and it appears that youth internalise their experiences with their attachment figures, and are better able to use these symbolic representations as a source of personal strength or resilience, compared to older adults who rely on more external supports for coping with stress (Mikulincer et al., 2003). Thus, deficits in support seeking and giving skills or an overly avoidant pattern of behavior when under stress may not yet be detrimental to mental health. Further study is warranted to assess the developmental changes that may underpin this pathway of associations.

In contrast to the findings for emotion suppression, our results suggest that emotion dysregulation plays a substantial role in psychopathology, both directly and by channelling the negative impact of anxious attachment. Nevertheless, the rather small indirect effects, relative to the direct effects, in the model suggests that attachment and emotion dysregulation have unique features that each explain symptomatology among emerging adults, but that there may also be other antecedents of emotion dysregulation that could be considered when expanding on the model tested here. As such, future research, preferably longitudinal, should consider other social situations, social experiences and individual characteristics that may be foundations of the development of emotion dysregulation and result in the emergence or escalation of symptoms over time. The particular focus should be on what might predict sadness dysregulation rather than anger dysregulation and vice versus. One possibility is to focus on appraisals and attributions for negative events. For example, relying on self-blame when negative events occur could prompt more difficulties with internalized emotions such as sadness when compared to blaming others when negative events occur; other-blame might be more strongly associated with difficulty regulating externalized emotions, such as anger (Guerra, Asher, \& DeRosier, 2004; Sandstrom \& Coie, 1999; Taylor, Sullivan, \& Kliewer, 2013; Zimmer-Gembeck et al., 2016). 


\section{Future Research and Study Limitations}

Future research should continue to focus on attachment and the specific emotion regulation strengths and weaknesses that may covary with attachment orientations or may follow from them. Mikulincer and Shaver (2007) proposed how attachment orientations should be associated with ER in particular ways, but they (and many researchers in the field of ER) tend to refer to distress and emotions in general, rather than identifying the specific emotion that are subject to regulation. As described in theory (e.g., Campos et al., 1994) and supported with the current findings, ER may vary as a function of specific emotion type. This is somewhat congruent with the differential emotions perspective which assumes that each distress emotion has a distinct adaptive social function and elicits particular reactions from a social partner or from the social world more generally (Izard, 1991). Some descriptions of attachment theory also suggest that the experience of a specific emotion may differ and have different functions depending on an individual's attachment orientation goals. For example, the social function of anger might function to push others away or as an attempt to change the behavior of another person to maintain closeness (Bowlby, 1973; Mikulincer, 1998). This suggests that future research might not only focus on specific emotions, but might also need to try to capture the different functions of what might be assumed to be the same emotion. For example, trying to understand the meaning, display, and perceived function of anger for individuals high in anxious attachment as compared to those high in avoidant attachment.

Our investigation of the associations between attachment, ER and symptoms of psychopathology filled several gaps in the literature by examining social anxiety along with depressive symptoms and aggressive behavior, by examining regulation of two different emotions, and by studying the associations in emerging adults. Nevertheless, several limitations 
should be acknowledged. First, generalizability may be compromised because the sample was limited to Australian university students. However, university students in Australia are diverse in socioeconomic and cultural background, but can be slightly younger than university students in other parts of the world. Second, self-report was the only method of data collection. Social desirability may be a factor in reporting and shared method variance may have resulted in stronger associations in some cases.

\section{Conclusion}

In summary, the findings build on previous research (e.g., Brenning \& Braet, 2013) to show that attachment anxiety and avoidance, as well as emotion dysregulation, in emerging adulthood are associated with elevated self-reported symptoms of emotional and behavioral problems. Differentiated pathways are also suggested. First, individuals higher in anxious attachment report more sadness and anger dysregulation, whereas individuals higher in avoidant attachment report more suppression of sadness and anger. Second, sadness as compared to anger dysregulation exhibited a different pattern of associations with depression and social anxiety relative to aggressive behavior. Continued attention to the dysregulation of specific emotions is a useful direction for future developmental psychopathology research, as this may be one way to begin to identify common and unique risks for the emergence and continuation of different forms of psychopathology. Such an approach is consistent with many aspects of developmental psychopathology (Cicchetti \& Cohen, 1995) and attachment theory (Brenning \& Braet, 2013; Mikulincer \& Shaver, 2007, 2012; Zimmer-Gembeck et al., 2017), as well as being consistent with functionalist and differential emotion theories (e.g., Campos et al., 1994; Izard, 1991). 


\section{References}

Achenbach, T. M., \& Rescorla, L. A. (2001). Manual for ASEBA school-age forms and profiles. Burlington: University of Vermont, Research Centre for Children, Youth, \& Families.

Aldao, A., Nolen-Hoeksema, S., \& Schweizer, S. (2010). Emotion-regulation strategies across psychopathology: A meta-analytic review. Clinical Psychology Review, 30, 217 - 237. doi:10.1016/j.cpr.2009.11.004

Bowlby, J. (1969). Attachment and loss: Vol. 1. London, UK: Hogarth P.

Bowlby, J. (1973). Attachment and loss: Vol. 2. Separation: Anxiety and anger. New York: Basic Books.

Bowlby, J. (1980). Attachment and loss: Vol. 3. Sadness and depression. New York, NY: Basic Books.

Brenning, K. M., \& Braet, C. (2013). The emotion regulation model of attachment: An emotion specific approach. Personal Relationships, 20, 107-123. doi:10.111/j.14756811.2012.01399.x

Brenning, K. M., Soenens, B., Braet, C., \& Bosmans, G. (2012). Attachment and depressive symptoms in middle childhood and early adolescence: Testing the validity of the emotion regulation model of attachment. Personal Relationships, 19, 445-464.

doi:10.1111/j.1475-6811.2011.01372.x

Brumariu, L. E. (2015). Parent-child attachment and emotion regulation. In G. Bosmans \& K. A. Kerns (Eds.), Attachment in middle childhood: Theoretical advances and new directions in an emerging field. New Directions for Child and Adolescent Development, 148, 31-45. 
Brumariu, L. E., \& Kerns, K. A. (2013). Pathways to anxiety: Contributions of attachment history, temperament, peer competence, and ability to manage intense emotions. Child Psychiatry and Human Development, 44, 504-515. doi:10.1007/s10578-012-0345-7

Campos, J. J., Mumme, D. L., Kermoian, R., \& Campos, R. G. (1994). A functionalist perspective on the nature of emotion. Monographs of the Society for Research in Child Development, 59, 284-303. doi:10.2307/1166150

Cassidy, J. (1994). Emotion regulation: Influences of attachment relationships. Monographs of the Society for Research in Child Development, 59, 228-249. doi:10.2307/1166148

Cicchetti, D., \& Cohen, D. J. (Eds.). (1995). Developmental Psychopathology, Vol. 1: Theory and Methods. New York: Wiley.

Clear, S. J., \& Zimmer-Gembeck, M. J. (2017). Associations between attachment and emotionspecific emotion regulation with and without relationship insecurity priming. International Journal of Behavioral Development. doi:10.1177/0165025415620057

Compas, B. E., Connor-Smith, J. K., Saltzman, H., Thomsen, A. H., \& Wadsworth, M. E. (2001). Coping with stress during childhood and adolescence: Problems, progress, and potential in theory and research. Psychological Bulletin, 127, 87-127. doi:10.1037/00332909.127.1.87

Contreras, J. M., Kerns, K. A., Weimer, B. L., Gentzler, A. L., \& Tomich, P. L. (2000). Emotion regulation as a mediator of associations between mother-child attachment and peer relationships in middle childhood. Journal of Family Psychology, 14, 111-124. doi:10.1037/0893-3200.14.1.111 
Darcy, K., Davila, J., \& Beck, G. J. (2005). Is social anxiety associated with both interpersonal avoidance and interpersonal dependence. Cognitive Therapy and Research, 29, 171-186. doi:10.1007/s10608-005-3163-4

Emde, R. (1980). Toward a psychoanalytic theory of affect. Part 1. The organizational model and its propositions. In S. Greenspan \& G. Pollock (Eds.), The course of life: Psychoanalytic contributions toward understanding personality and development (pp. 63-83). Adelphi, MD: Mental Health Study Center, NIMH.

Guerra, V. S., Asher, S. R.,\& DeRosier, M. E. (2004). Effect of children's perceived rejection on physical aggression. Journal of Abnormal Child Psychology, 32, 551-563. doi:10.1023/B:JACP.0000037783.88097.69.

Haga, S. M., Kraft, P., \& Corby, E. (2009). Emotion regulation: Antecedents and well-being outcomes of cognitive reappraisal and expressive suppression in cross-cultural samples. Journal of Happiness Studies, 10, 271 - 297. doi:10.1007/s10902-007-9080-3

Izard, C. E. (1991). The psychology of emotions. New York: Plenum.

Kobak, R., Cassidy, J., Lyons-Ruth, K., \& Ziv, Y. (2006). Attachment, stress, and psychopathology: A developmental pathways model. In D. Cicchetti \& D. J. Cohen (Eds.), Developmental psychopathology (pp. 333-369). New Jersey: John Wiley \& Sons.

Kopp, C. B. (1989). Regulation of distress and negative emotions: A developmental view. Developmental Psychology, 25, 343-353. doi: 10.1037/0012-1649.25.3.343

Kerns, K. A. (2008). Attachment in middle childhood. In J. Cassidy \& P. R. Saver (Eds.), Handbook of attachment: Theory, research, and clinical applications (2nd ed., pp. 366382). New York: Guilford Press. 
Kullik, A., \& Petermann, F. (2013). Attachment to parents and peers as a risk factor for adolescent depressive disorders: The mediating role of emotion regulation. Child Psychiatry and Human Development, 44, 537-548. doi:10.1007/s10578-012-0347-5

La Greca, A. M., \& Lopez, N. (1998). Social anxiety among adolescents: Linkages with peer relations and friendships. Journal of Abnormal Child Psychology, 26, 83-84. doi:10.1023/A:1022684520514

Lyons-Ruth, K., \& Jacobvitz, D. (2008). Attachment disorganization: Genetic factors, parenting contexts, and developmental transformation from infancy to adulthood. Handbook of Attachment: Theory, Research and Clinical Applications (pp. 666-697) New York: Guilford Press.

Malik, S., Wells, A., \& Wittkowski, A. (2015). Emotion regulation as a mediator in the relationship between attachment and depressive symptomatology: A systematic review. Journal of Affective Disorders, 172, 428-444. doi:10.1016/j.jad.2014.10.007.

Marganska, A., \& Gallagher, M., \& Miranda, R. (2013). Adult attachment, emotion dysregulation, and symptoms of depression and generalized anxiety disorder. American Journal of Orthopsychiatry, 83, 131-141. doi:10.1111/ajop.12001.

Mikulincer, M. (1998). Adult attachment style and individual differences in functional versus dysfunctional experiences of anger. Journal of Personality and Social Psychology, 74, 513-524. doi:10.1037/0022-3514.74.2.513

Mikulincer, M., \& Shaver, P. R. (2007). Attachment in adulthood: Structure, dynamics, and change. New York, NY: Guilford.

Mikulincer, M., \& Shaver, P. R. (2012). An attachment perspective on psychopathology. World Psychiatry, 11, 11-15. 
Mikulincer, M., Shaver, P. R., \& Pereg, D. (2003). Attachment theory and affect regulation: The dynamics, development, and cognitive consequences of attachment-related strategies. Motivation and Emotion, 27, 77-102. doi:10.1023/A:1024515519160

Morris, A. S., Silk, J. S., Steinberg, L., Myers, S. S., \& Robinson, L. R. (2007). The role of the family context in the development of emotion regulation. Social Development, 16, 361388. doi: 10.1111/j.1467-9507.2007.00389.x.

Muris, P., Meesters, C., van Melick, M., \& Zwambag, L. (2001). Self-reported attachment style, attachment quality, and symptoms of anxiety and depression in young adolescents. Personality and Individual Differences, 30, 809-818. doi:10.1016/S0191-8869(00)00074$\mathrm{X}$

Newman, K. M. (2013). A more usable Winnicott. Psychonalytic Inquiry, 33, 59-68. doi:10.1080/07351690.2013.743805.

Roth, G., Assor, A., Niemiec, C. P., Ryan, R. M., \& Deci, E. L. (2009). The emotional and academic consequences of parental conditional regard: Comparing conditional positive regard, conditional negative regard, and autonomy support as parenting practices. Developmental Psychology, 45, 1119-1142. doi:10.1037/a0015272

Sandstrom, M. J., \& Coie, J. D. (1999). A developmental perspective on peer rejection: mechanisms of stability and change. Child Development, 70, 955-966. doi:10.1111/14678624.00069 .

Seiffge-Krenke, I. (2011). Coping with relationship stressors: A decade review. Journal of Research on Adolesence, 21 196-210. doi:10.1111/j.1532-7795.2010.00723.x

Shaver, P. R., \& Mikulincer, M. (2002). Attachment related psychodynamics. Attachment and Human Development, 4, 133-161. doi:10.1080/14616730210154171 
Skinner, E. A., \& Zimmer-Gembeck, M. J. (2007). The development of coping. Annual Review of Psychology, 58, 119-144.

Skinner, E. A., \& Zimmer-Gembeck, M. J. (2016). The development of coping from birth to emerging adulthood: Neurophysiological and social underpinnings, qualitative shifts, and differential pathways towards psychopathology and resilience. New York: Springer.

Southam-Gerow, M. A., \& Kendall, P. C. (2002). Emotion regulation and understanding: Implications for child psychopathology and therapy. Clinical Psychology Review, 22, 189-222. doi:10.1016/S0272-7358(01)00087-3

Sroufe, L. A. (1995). Emotional development: The organization of emotional life in the early years. New York: Cambridge University Press.

Taylor, K. A., Sullivan, T. N., \& Kliewer, W. (2013). A longitudinal path analysis of peer victimization, threat appraisals to the self, and aggression, anxiety, and depression among urban African American adolescents. Journal of Youth and Adolescence, 42, 178-189. doi:10.1007/s10964-012-9821-4.

Viana, A. G., \& Rabian, B. (2008). Perceived attachment: Relations to anxiety sensitivity, worry, and GAD symptoms. Behavior Research and Therapy, 46, 737-747. doi:10.1016/j.brat.2008.03.002

Wei, M., Heppner, P. P., \& Mallinckrodt, B. (2003). Perceived coping as a mediator between attachment and psychological distress: A structural equation modeling approach. Journal of Counseling Psychology, 50, 438-447. doi:10.1037/0022-0167.50.4.438

Wei, M., Vogel, D. L., Ku, T.-Y., \& Zakalik, R. A. (2005). Adult attachment, affect regulation, negative mood, and interpersonal problems: The mediating roles of emotional reactivity 
and emotional cutoff. Journal of Counselling Psychology, 52, 14-24. doi:10.1037/00220167.52 .1 .14

Wilkinson, R. B. (2011). Measuring attachment dimensions in adolescents: Development and validation of the experiences in close relationships- Revised- General short form. Journal of Relationships Research, 2, 53-62. doi:10.1375/jrr.2.1.53

Winnicott, D. W. (1965). The maturational processes and the facilitating environment. New York: International Universities Press.

Zimmer-Gembeck, M. J., Nesdale, D., Webb, H. J., Khatibi, M., \& Downey, G. (2016). A longitudinal rejection sensitivity model of depression and aggression: Unique roles of anxiety, anger, blame, withdrawal, and retribution. (2016). Journal of Abnormal Child Psychology, 44, 1291-1307. doi:10.1007/s10802-016-0127-y.

Zimmer-Gembeck, M. J, \& Skinner, E. A. (2016). The development of coping and regulation: Implications for psychopathology and resilience. In D. Cicchetti (Ed.) Developmental psychopathology (3rd Ed., Vol. 4, pp. 485-544). New York: Wiley. http://au.wiley.com/WileyCDA/WileyTitle/productCd-1118121791.html

Zimmer-Gembeck, M. J., Webb, H. J., Pepping, C. A., Swan, K., Merlo, O., Skinner, E. A., Avdagic, E., \& Dunbar, M. (2017). Review: Caregiver-child attachment, emotion regulation, and coping with stress. International Journal of Behavioral Development, 4, 74-93. doi:10.1177/0165025415618276 
Table 1

Correlations between All Measures $(N=\mathbf{3 8 3})$

\begin{tabular}{|c|c|c|c|c|c|c|c|c|}
\hline Measure & 1 & 2 & 3 & 4 & 5 & 6 & 7 & 8 \\
\hline 1. Attachment Anxiety & --- & & & & & & & \\
\hline 2. Attachment Avoidance & $.37 * *$ & --- & & & & & & \\
\hline 3. Depressive symptoms & $.45 * *$ & $.30 * *$ & --- & & & & & \\
\hline 4. Social Anxiety symptoms & $.60 * *$ & $.31 * *$ & $.52 * *$ & --- & & & & \\
\hline 5. Aggressive behavior & $.36^{* *}$ & $.15^{* *}$ & $.51 * *$ & $.44 * *$ & --- & & & \\
\hline 6. Sad dysregulation & $.43 * *$ & $.17 * *$ & $.47 * *$ & $.42 * *$ & $.27 * *$ & --- & & \\
\hline 7. Sad suppression & $.22 * *$ & $.39 * *$ & $.19 * *$ & $.14^{* *}$ & $.15 * *$ & $.20 * *$ & --- & \\
\hline 8. Anger dysregulation & $.34 * *$ & $.13 *$ & $.36 * *$ & $.36^{* *}$ & $.42 * *$ & $.56^{* *}$ & $.16^{* *}$ & --- \\
\hline 9. Anger suppression & $.11 *$ & $.12 *$ & .08 & $.12 *$ & .04 & $.18 * *$ & $.43 * *$ & .04 \\
\hline
\end{tabular}

$* p<.05$. ** $p<.01$. 


\section{Table 2}

Means and SDs for All Participants and for Young Men and Women Separately, and Tests of Sex Differences $(N=383)$

\begin{tabular}{lcccc}
\hline & Overall & Young Men & Young Women & \\
Measure & $M(S D)$ & $\begin{array}{c}M(S D) \\
n=181\end{array}$ & $\begin{array}{c}M(S D) \\
n=202\end{array}$ & $t(1,381)$ \\
\hline Anxious attachment & $2.77(0.70)$ & $2.77(0.71)$ & $2.77(0.70)$ & 0.02 \\
Avoidant attachment & $2.87(0.60)$ & $2.85(0.55)$ & $2.89(0.64)$ & -0.57 \\
Depressive symptoms & $0.49(0.44)$ & $0.45(0.44)$ & $0.55(0.43)$ & $-2.29 *$ \\
Social anx symptoms & $2.33(0.81)$ & $2.24(0.81)$ & $2.41(0.81)$ & $-2.02^{*}$ \\
Aggressive behavior & $1.64(0.58)$ & $1.76(0.66)$ & $1.53(0.47)$ & $4.05^{* *}$ \\
Sad dysregulation & $2.65(0.91)$ & $2.46(0.85)$ & $2.82(0.92)$ & $-3.98^{* *}$ \\
Sad suppression & $3.00(0.84)$ & $3.16(0.93)$ & $3.17(0.88)$ & -0.03 \\
Anger dysregulation & $2.75(0.92)$ & $2.41(0.94)$ & $2.44(0.89)$ & -0.31 \\
Anger suppression & $3.05(0.86)$ & $3.10(0.89)$ & $3.00(0.83)$ & 1.19 \\
\hline
\end{tabular}

$* p<.05 . * * p<.01$. 
Table 3

Standardized Associations of Attachment and Emotion Regulation with Symptoms and Aggression (Paths A in Figure 1, see also Figure 2 for an illustration) $(N=383)$

Emotion Regulation

\begin{tabular}{lllll} 
Attachment & Sad Dysregulation & Sad Suppression & Anger Dysregulation & Anger Suppression \\
\hline Anxious & $.42^{* *}$ & .09 & $.34^{* *}$ & .07 \\
Avoidant & .01 & $.36^{* *}$ & .00 & $.10^{*}$ \\
\hline
\end{tabular}

${ }^{*} p<.05 .{ }^{* *} p<.01$; based on bootstrap bias-corrected confidence intervals, and associated 95\% confidence intervals and $p$-values.

$\chi 2(1)=0.00, p=.99, \mathrm{CFI}=1.00, \mathrm{RMSEA}=.000(90 \% \mathrm{CI}=.000-.000), p=1.00$. 


\section{Table 4}

Standardized Direct, Indirect, and Total Associations of Attachment and Emotion Regulation on Symptoms and Aggression (see also Figure 2 for an illustration of the direct associations, $N=383$ )

\begin{tabular}{|c|c|c|c|c|c|c|c|c|c|}
\hline \multirow[b]{2}{*}{ Predictors } & \multicolumn{3}{|c|}{$\begin{array}{l}\text { Direct Associations } \\
\text { (Paths B and } \\
\text { C, Fig. 1) } \\
\end{array}$} & \multicolumn{3}{|c|}{$\begin{array}{c}\text { Total Indirect Associations } \\
\text { via all ER Measures } \\
(\text { Paths } A \times B \text {, Fig. 1) })^{1} \\
\end{array}$} & \multicolumn{3}{|c|}{$\begin{array}{c}\text { Total Associations } \\
\text { Total Associations } \\
(\mathrm{C}+\mathrm{A} \times \mathrm{B} \text { Paths, Fig. } 1) \\
\end{array}$} \\
\hline & Dep & SA & Agg & Dep & SA & Agg & Dep & SA & Agg \\
\hline Anxious & $.21 * *$ & $.45^{* *}$ & $.26^{* *}$ & $.18^{* *}$ & $.10^{* *}$ & $.11^{* *}$ & $.39 * *$ & $.55^{* *}$ & $.37 * *$ \\
\hline Avoidant & $.16^{* *}$ & $.13^{*}$ & .00 & .01 & -.02 & .02 & $.17^{* *}$ & .11 & .02 \\
\hline Sad dysregulation & $.31 * *$ & $.15^{*}$ & -.03 & -- & -- & -- & $.31 * *$ & $.15^{*}$ & -.03 \\
\hline Sad suppression & .02 & -.08 & .07 & -- & -- & -- & .02 & -.08 & .07 \\
\hline Anger dysregulation & .12 & .12 & $.35^{* *}$ & -- & -- & -- & .12 & .12 & $.35^{* *}$ \\
\hline Anger suppression & -.01 & .06 & -.02 & -- & -- & -- & -.01 & .06 & -.02 \\
\hline $\begin{array}{l}{ }^{*} p<.05 .{ }^{*} p<.01 ; \mathrm{b} \\
\chi 2(1)=0.00, p=.99 \\
\text { Dep }=\text { depression. SA } \\
\text { See Table } 3 \text { for the est } \\
{ }^{1} \text { See text for a descrip } \\
\text { ER (i.e., sadness dysre }\end{array}$ & $\begin{array}{l}\mathrm{d} \text { on bo } \\
\mathrm{FI}=1.0 \\
\text { social a } \\
\text { tates for } \\
\text { n of the }\end{array}$ & $\begin{array}{l}\text { strap bi } \\
\text { RMSE } \\
\text { iety. A } \\
\text { aths A } \\
\text { gnifica } \\
\text { dness st }\end{array}$ & $\begin{array}{l}\text { s-correct } \\
=.000 \\
g=\text { aggre } \\
\text { Figure } \\
\text { t indirect } \\
\text { ppressior }\end{array}$ & $\begin{array}{l}\text { fidence } \\
\text { CI }=.00 \\
\text { behavic }\end{array}$ & $\begin{array}{l}\text { vals an } \\
0), p= \\
\text { s }=\text { dys } \\
\text { achmen } \\
\text { on, and }\end{array}$ & ciated & uppressic & 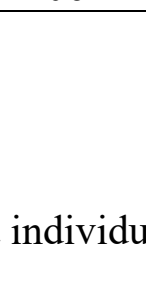 & \\
\hline
\end{tabular}




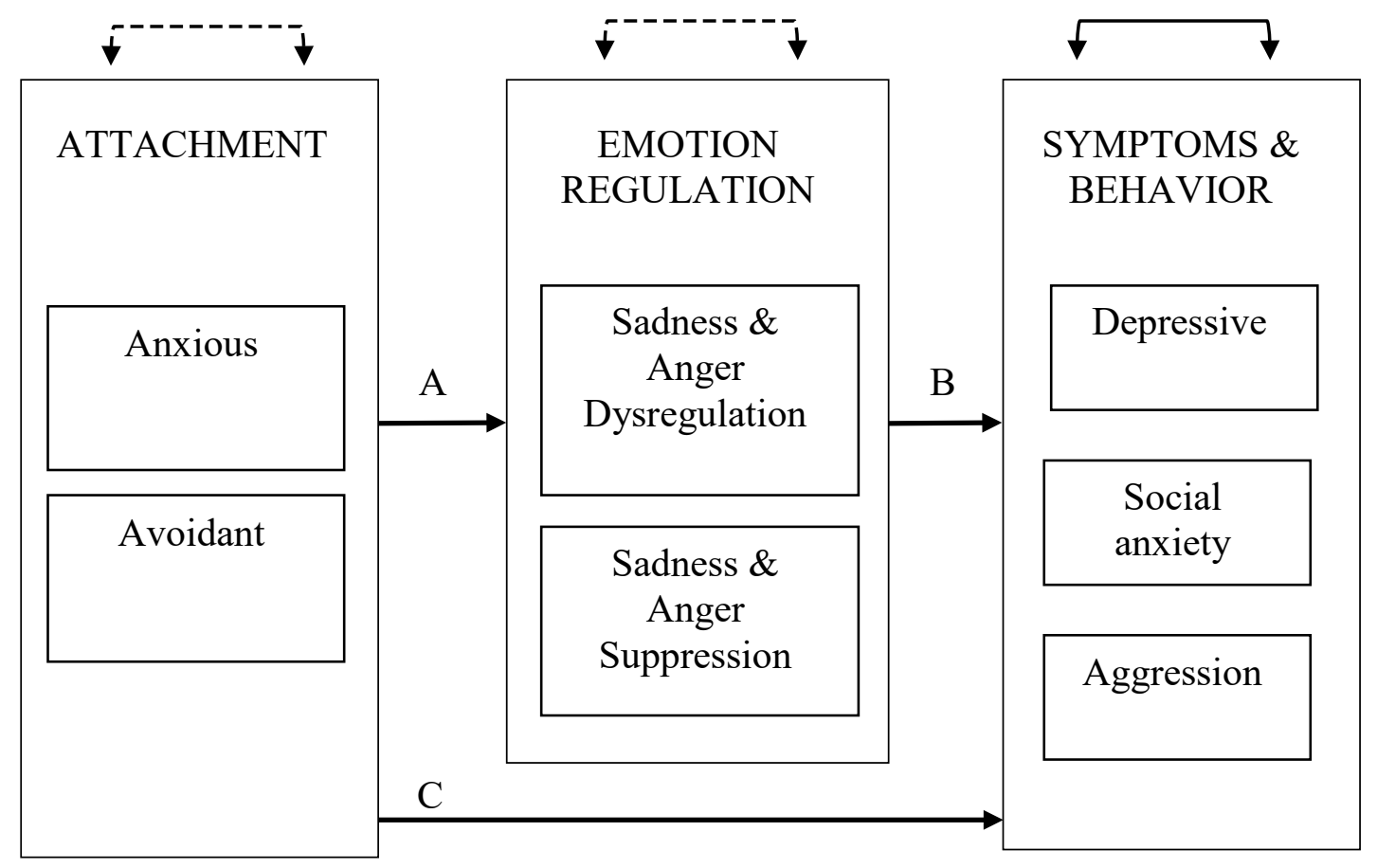

Figure 1. An illustration of the tested model.

Note. Solid directional arrows (A, B, and C) between sets of measures indicate that all paired associations were estimated in the path model. The solid two-headed arrow indicates that all covariances within a set of measures were freed. The hatched two-headed arrows indicate that covariances between attachment classifications and between emotion regulation subscales were freed with the exception of one nonsignificant $(p>.10)$ association. 


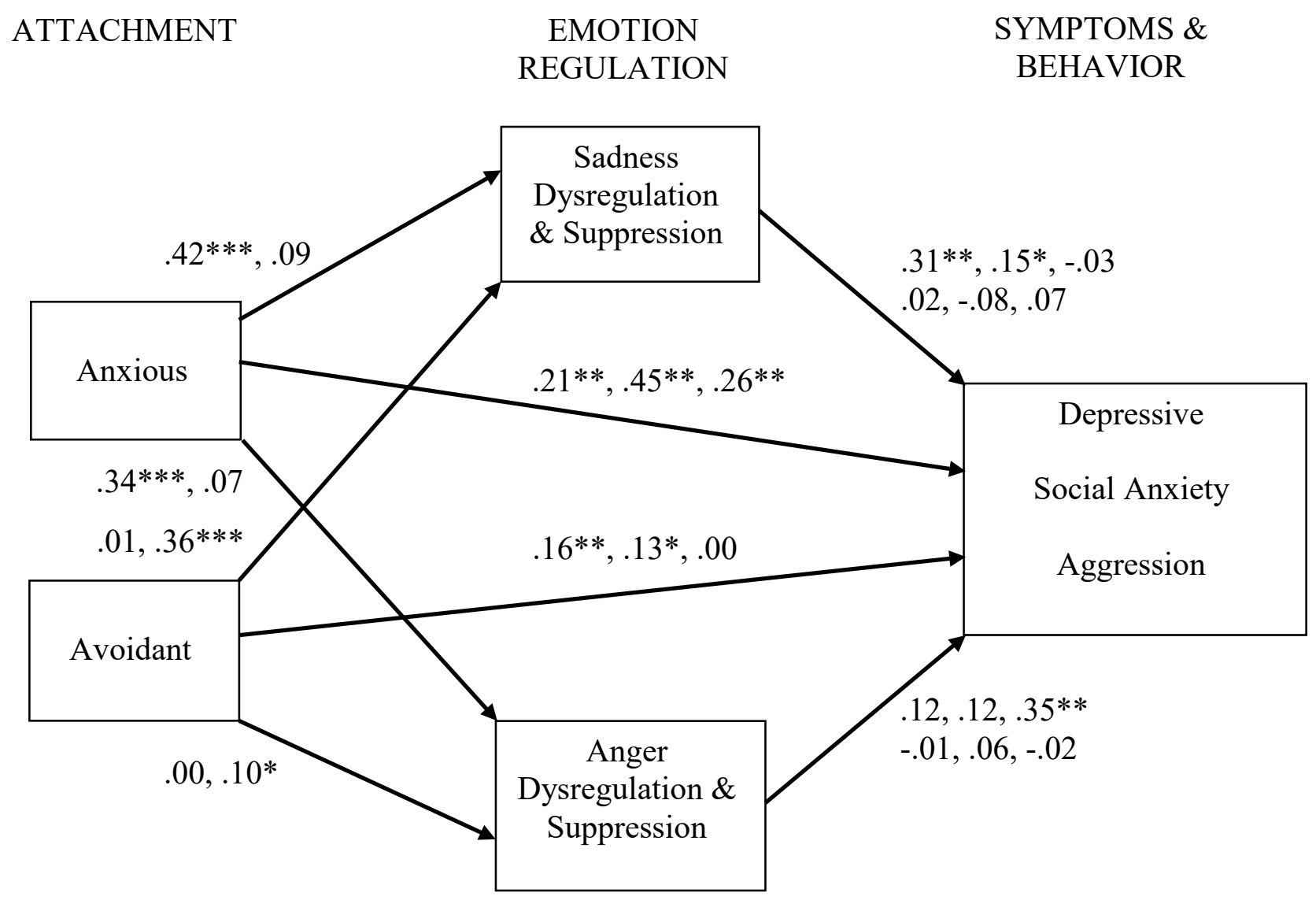

Figure 2. An illustration of the standardized direct effects in the final model.

Note. Multiple path estimates are shown on each path. For example, the path estimates on the path from anxious attachment to sadness dysregulation \& suppression show the effect of anxious attachment on dysregulation followed by the effect of anxious attachment on suppression. Also, for example, for the path estimates from emotion regulation to symptoms, the first three path estimates are the effects of sadness dysregulation on each of the symptom measures (depression, social anxiety, and aggression); the second three path estimates are the effects of sadness suppression on each of the symptoms, respectively. 